\title{
Torque Density Improvement in a Six-Phase Induction Motor With Third Harmonic Current Injection
}

\author{
Renato O. C. Lyra, Member, IEEE, and Thomas A. Lipo, Fellow, IEEE
}

\begin{abstract}
The use of six-phase induction motor for industrial drives presents several advantages over the conventional three-phase drive such as improved reliability, magnetic flux harmonic reduction, torque pulsations minimization, and reduction on the power ratings for the static converter. For these reasons, six-phase induction motors are beginning to be a widely acceptable alternative in high power applications. A typical construction of such drives includes an induction machine with a dual three-phase connection, where two three-phase groups are spatially shifted 30 electrical degrees, a six-leg inverter, and a control circuit. By controlling the machine's phase currents, harmonic elimination and torque-ripple reduction techniques could be implemented. This paper describes a technique of injecting third harmonic zero sequence current components in the phase currents, which greatly improves the machine torque density. Analytical, finite-element, and experimental results are presented to show the system operation and to demonstrate the improvement on the torque density.
\end{abstract}

Index Terms-Induction machine analysis and design, industrial drives, six-phase drives.

\section{INTRODUCTION}

$\mathbf{T}$ HREE-PHASE induction machines are today a standard for industrial electrical drives. Cost, reliability, robustness, and maintenance-free operation are among the reasons these machines are replacing dc drive systems. The development of power electronics and signal processing systems has eliminated one of the greatest disadvantages of such ac systems, that is, the issue of control. With modern techniques of field-oriented vector control, the task of variable-speed control of induction machines is no longer a disadvantage.

The need to increase system performance, particularly when facing limits on the power ratings of power supplies and semiconductors, motivates the use of phase number other than three, and encourages new pulsewidth modulation (PWM) techniques, new machine design criteria, and the use of harmonic current and flux components.

Paper IPCSD 02-027, presented at the 2001 Industry Applications Society Annual Meeting, Chicago, IL, September 30-October 5, and approved for publication in the IEEE TRANSACTIONS ON INDUSTRY APPLICATIONS by the Industrial Drives Committee of the IEEE Industry Applications Society. Manuscript submitted for review October 15, 2001 and released for publication May 29, 2002. This work was supported by the WEMPEC Consortium at the University of Wisconsin, Madison, the Universidade Federal de Minas Gerais, Brazil, and Coordenação de Aperfeiçoamento de Pessoal de Nível Superior, Brazil.

R. O.C. Lyra was with the Department of Electric and Computer Engineering, University of Wisconsin, Madison, WI 53706 USA. He is now with the Department of Electric and Computer Engineering, Universidade Federal de Minas Gerais, Belo Horizonte, Brazil (e-mail: lyra@ ieee.org).

T. A. Lipo is with the Department of Electric and Computer Engineering, University of Wisconsin, Madison, WI 53706 USA (e-mail: lipo@engr.wisc.edu).

Publisher Item Identifier 10.1109/TIA.2002.802938.
In a multiphase system, here assumed to be a system that comprises more than the conventional three phases, the machine output power can be divided into two or more solid-state inverters that could each be kept within prescribed power limits. Also, having additional phases to control means additional degrees of freedom available for further improvements in the drive system.

With split-phase induction machines, and appropriate drive system, the sixth harmonic torque pulsation, typical in a six-step three-phase drive, can be eliminated [1], [2]. Also, air-gap flux created by fifth and seventh harmonic currents in a high-power six-step converter-fed system is dramatically reduced with the penalty of increased converter harmonic currents [3]. PWM techniques are employed to overcome this problem by eliminating the harmonic currents in the modulation process when the power ratings are not prohibitive.

Dual-stator machines are similar to split-phase machines with the difference that the stator groups are not necessarily equal. A dual-stator machine with different numbers of poles in each three-phase group has been proposed in [4] to obtain controllability at low speeds. Two independent stator windings are used in [5] for an induction generator system. One set of windings is responsible for the electromechanical power conversion while the second one is used for excitation purposes. A PWM converter is connected to the excitation windings and the load is connected directly to the power windings.

A particular case of split-phase or dual-stator machine, the six-phase machine can be built by splitting a three-phase winding into two groups. Usually these three-phase groups are displaced by 30 electrical degrees from each other. This arrangement composes an asymmetrical six-phase machine since the angular distance between adjacent phases is not all the same [6]. The analysis of an induction machine for multiple phases and arbitrary displacement between them is presented in [2] where the six-phase induction machine is used as an example and an equivalent circuit has been derived. The $d q 0$ model for a six-phase machine was developed in [7].

Reliability is one of the advantages in using six-phase systems. In the case of failure of one of the phases, either in the machine or in the power converter, the system can still operate at a lower power rating since each three-phase group can be made independent from each other. In the case of losing one phase, the six-phase machine can continue to be operated as a five-phase machine as described in [8].

The inherent third harmonic component in the winding functions of the machine [9], [10] suggests the use of third harmonic currents to improve its performance. Torque improvement can be obtained by using multiphase windings with injection of 
third harmonic currents. Such a nine-phase induction machine drive was investigated in [11]. The complexity of the power system, which includes series and parallel transformers, increases the system cost and must be evaluated for each particular application. The use of a voltage-controlled system does not guarantee the phase alignment between fundamental and third harmonic currents, especially at low speeds, and a poor low speed and dynamic behavior can be expected for this system.

This paper describes a technique of injecting third harmonic, zero-sequence current components in the phase currents that improve the machine torque density. Analytical, finite-element, and experimental results are presented to show the system operation and to demonstrate the improvement on the torque density.

\section{TORQUE IMPROVEMENT WITH THIRD HARMONIC CURRENT INJECTION}

The asymmetric six-phase machine is derived from a conventional three-phase induction machine. For a two-pole concentrated-winding three-phase machine, the magnetomotive force (MMF) acting across the air gap associated with the stator currents is

$$
F=F_{a}+F_{b}+F_{c}=N_{a}(\phi) i_{a}+N_{b}(\phi) i_{b}+N_{c}(\phi) i_{c}
$$

where $N_{a}, N_{b}$, and $N_{c}$ are the machine's winding functions [12] and compose a set of $120^{\circ}$ phase-shifted square waves with amplitude $2 N_{s}$ (each coil has $4 N_{s}$ turns). Using Fourier analysis decomposition, and assuming balanced three-phase currents with amplitude $I$, it can be shown that the even and all triple harmonics are zero and the MMF is given by

$$
F=F_{1}+F_{5}+F_{7}+\cdots
$$

where

$$
\begin{aligned}
& F_{1}=\left(\frac{3}{2}\right)\left(\frac{4}{\pi}\right)\left(2 N_{s}\right) I[\sin (\phi-\theta)] \\
& F_{5}=\left(\frac{1}{5}\right)\left(\frac{3}{2}\right)\left(\frac{4}{\pi}\right)\left(2 N_{s}\right) I[\sin (5 \phi-\theta)] \\
& F_{5}=\left(\frac{1}{7}\right)\left(\frac{3}{2}\right)\left(\frac{4}{\pi}\right)\left(2 N_{s}\right) I[\sin (7 \phi-\theta)]
\end{aligned}
$$

and the wave contains a $0 \%$ third harmonic, $20 \%$ fifth harmonic, and $14 \%$ seventh harmonic, plus smaller portions of higher harmonics. Setting the sine function argument equal to a constant, to establish a fixed point in the waveform, and differentiating with respect to time, the rotational speed can be calculated. The peak fundamental component rotates in the direction of increasing $\phi$ with angular speed $\omega$, the fifth harmonic rotates in the direction of decreasing $\phi$ at $1 / 5$ the speed of the fundamental component and the seventh harmonic rotates at the same direction of the fundamental with $1 / 7$ of its speed. The fifth harmonic produces a negative-sequence component of flux that produces negative or braking torque. The seventh produces positive torque but it is only useful between $0-1 / 7$ of the synchronous speed.

If a neutral connection is provided, zero-sequence current components can flow in the machine. Considering a zero- sequence current $I_{0}$ the MMF due to this current can be calculated to be

$$
F_{0}=\left(\frac{4}{\pi}\right)\left(2 N_{s}\right)\left(\frac{1}{3} \sin (3 \phi)+\frac{1}{9} \sin (9 \phi)+\cdots\right) I_{0}
$$

where the triple harmonic of the square-wave winding functions are clearly represented. This suggests the use of triplens of the fundamental current frequency to produce torque corresponding to the zero sequence winding functions. Since most of the machines have a discrete distributed winding, the zero-sequence winding function is nonzero and can be explored to produce extra torque. Injecting a third harmonic current component $I_{0}=$ $I_{3} \sin (3 \theta)$ and neglecting higher harmonics, the MMF is

$$
F_{0}=\left(\frac{4}{\pi}\right)\left(2 N_{s}\right) \frac{I_{3}}{3}(\sin (3 \phi) \sin (3 \theta)) .
$$

This quantity represents a standing or pulsating wave in the air gap and not a rotating wave. This component is undesirable since it produces braking and pulsating torques.

Distributing the windings over more slots can reduce the harmonic content of the MMF. For a distribution over two slots, or two slots per pole per phase, the new fundamental MMF is

$$
F_{1}=\left(\frac{3}{2}\right)\left(\frac{4}{\pi}\right) N_{s} I\left[\sin (\phi-\theta)+\sin \left(\phi-\frac{\pi}{6}-\theta\right)\right] .
$$

This result corresponds to a three-phase machine with distributed windings or to a six-phase concentrated winding machine. Two three-phase winding groups, spatially phase shifted by $30^{\circ}$, compose the six-phase machine.

The amplitude of the fundamental component is, then,

$$
\left|F_{1}\right|=\left(\frac{3}{2}\right)\left(\frac{4}{\pi}\right) 2 N_{s} I\left[\frac{\sqrt{2+\sqrt{3}}}{2}\right]=k_{d 1}\left(\frac{3}{2}\right)\left(\frac{4}{\pi}\right) 2 N_{s} I
$$

and the constant $k_{d 1}=0.9593$, as expected, is the usual distribution factor for two slots per pole per phase. The fundamental component is therefore reduced by $4.1 \%$ when compared to the no-phase-shift full-pitch case. Doing similar analysis for the fifth and seventh harmonics, it can be shown that with the distribution over two slots, the fifth harmonic component is reduced from $20 \%$ to $5 \%$ and the seventh harmonic goes from $14 \%$ to $3.7 \%$. For this case, if a third harmonic zero-sequence component is injected, it would again produce a standing wave and is of no practical value again.

If, however, two sets of three-phase currents phase are shifted in time by $30^{\circ}$ to comprise the currents in the six-phase machine, the fundamental component of the MMF can be found to be the same as in the concentrated winding machine (3) but now both fifth and seventh harmonics are reduced to zero. With injection of third harmonic zero-sequence current components as

$$
I_{0, a b c}=I_{3} \cos (3 \theta) \quad \text { and } \quad I_{0, x y z}=I_{3} \sin (3 \theta)
$$

where $a b c$ and $x y z$ correspond to the two three-phase winding groups, the zero-sequence MMF is now

$$
F_{0}=\left(\frac{4}{\pi}\right) \frac{N_{s}}{3} I_{3}\{\sin [3(\phi-\theta)]\} .
$$




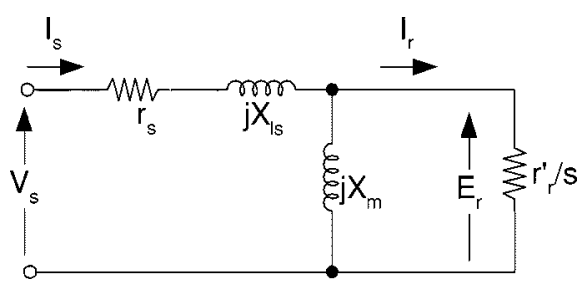

Fig. 1. Approximated equivalent circuit of a three-phase induction motor where the rotor leakage inductance is neglected.

This result corresponds to a rotating field with angular speed equal to the fundamental angular speed. Hence, the zero-sequence component can be now used to produce a second positively rotating flux component synchronized with the fundamental component.

\section{A. Flux Distribution With Third Harmonic Current Injection}

The possibility of injecting third harmonic current components into the machine without producing pulsating torques enables the ability to reshape the machine's flux distribution in a similar manner to the technique applied in PWM modulators. In PWM modulators, a third harmonic voltage reference is added to the fundamental component to increase the modulation index beyond the unity modulation index without distortion produced by dropping pulses. For the equivalent approach applied to the modulating machine flux, it is desired to increase the fundamental component of flux without saturating the machine.

The appropriate target reference flux waveform, containing the third harmonic contribution, is defined as

$$
B_{g}(\phi)=B_{1} \sin (\phi)+B_{3} \sin (3 \phi) .
$$

Using an optimization process, the relation between fundamental and third harmonic components can be determined for the best iron utilization and the air-gap flux is defined as a function of the maximum allowed flux distribution $B_{\max }$ as

$$
B_{g}(\phi)=\frac{2}{\sqrt{3}} B_{\max }\left[\sin (\phi)+\frac{1}{6} \sin (3 \phi)\right] .
$$

From (13), it is clear that for keeping the same peak value for the flux density in the air gap, with injection of the third harmonic component the peak of the fundamental flux component is higher.

\section{B. Torque Improvement With Third Harmonic Current Injection}

The benefit of using the third harmonic component can now be investigated. Using an approximate equivalent circuit for the induction machine, as shown in Fig. 1, where the rotor leakage inductance is neglected, and assuming peak values for the variables, the rotor current is

$$
I_{r}=\frac{E_{r} s}{r_{r}^{\prime}}
$$

and the torque is defined by

$$
T=\left(\frac{3}{2}\right)\left(\frac{P}{2}\right) \frac{E_{r}^{2} s}{\omega_{e} r_{r}^{\prime}} .
$$

The peak voltage $E_{r}$ is proportional to the peak air-gap flux density [12] by

$$
E_{r}=\omega_{e}\left(\frac{2}{\pi} B_{\max }\right) A_{\text {pole }} N_{s}
$$

where $A_{\text {pole }}$ is the area of one magnetic pole and $N_{s}$ is the number of series connected turns. The factor $2 / \pi$ expresses the average value of $B$ in terms of its peak value.

In the case of the asymmetric six-phase machine, the maximum allowable fundamental flux density can be increased by $2 / \sqrt{3}$. Since all other parameters in (15) remain the same for this machine, the increase in torque obtained by raising the fundamental component of flux density, while keeping the same peak tooth and air-gap flux density is

$$
\frac{T_{6 \text { phase-fund }}}{T_{\text {baseline }}}=\left(\frac{2}{\sqrt{3}}\right)^{2}=1.33
$$

where $T_{\text {baseline }}$ corresponds to the torque production in a threephase induction machine used as baseline for the evaluation. There is an additional $33 \%$ in the torque production for the six-phase machine with third harmonic injection due to the increase in the fundamental flux. In addition to that, the contribution of the third harmonic component must be considered. The torque produced by the third harmonic currents in the six-phase machine is

$$
T_{6 \text { phase, } 3 h}=3 \frac{P}{2} \frac{E_{r 3 h}^{2} s_{3 h}}{3 \omega_{e} r_{r 3 h}^{\prime}}
$$

and the third harmonic voltage can be computed as

$E_{r 3 h}=3 \omega_{e}\left(\frac{2}{\pi} B_{3}\right)\left(\frac{A_{\text {pole }}}{3}\right) 3\left(\frac{N_{s}}{3}\right)=\omega_{e}\left(\frac{2}{\pi} B_{3}\right) A_{\text {pole }} N_{s}$

where $B_{3}=(2 / \sqrt{3})(1 / 6) B_{\max }$.

The slip for the third harmonic is

$$
s_{3 h}=\frac{3 \omega_{e}-\left(\frac{3 P}{2}\right) \omega_{r m}}{3 \omega_{e}}=\frac{\omega_{e}-\left(\frac{P}{2}\right) \omega_{r m}}{\omega_{e}}
$$

and the rotor resistance can be determined [13] to be

$$
r_{r 3 h}^{\prime}=\frac{3(12)\left(\frac{N_{s}}{3}\right)^{2}}{S_{r}} r_{b e}
$$

where $N_{s} / 3$ is the number of series connected turns of one of the three pairs of poles of the third harmonic, $S_{r}$ is the number of rotor slots, and $r_{b e}$ is the resistance of a rotor bar taking into account the effect of the end ring. The factor of 3 is used since the three pole pairs of the third harmonic are connected in series. Inserting these expressions in the torque equation for the third harmonic (18)

$$
T_{6 \text { phase- } 3 h}=3\left(\frac{P}{2}\right) \frac{\left[\frac{1}{3 \sqrt{3}}\left(\frac{2}{\pi} B_{\max }\right) A_{\text {pole }}\right]^{2}\left(\omega_{e}-\frac{P}{2} \omega_{r m}\right)}{\frac{12}{S_{r}} r_{b e}} .
$$




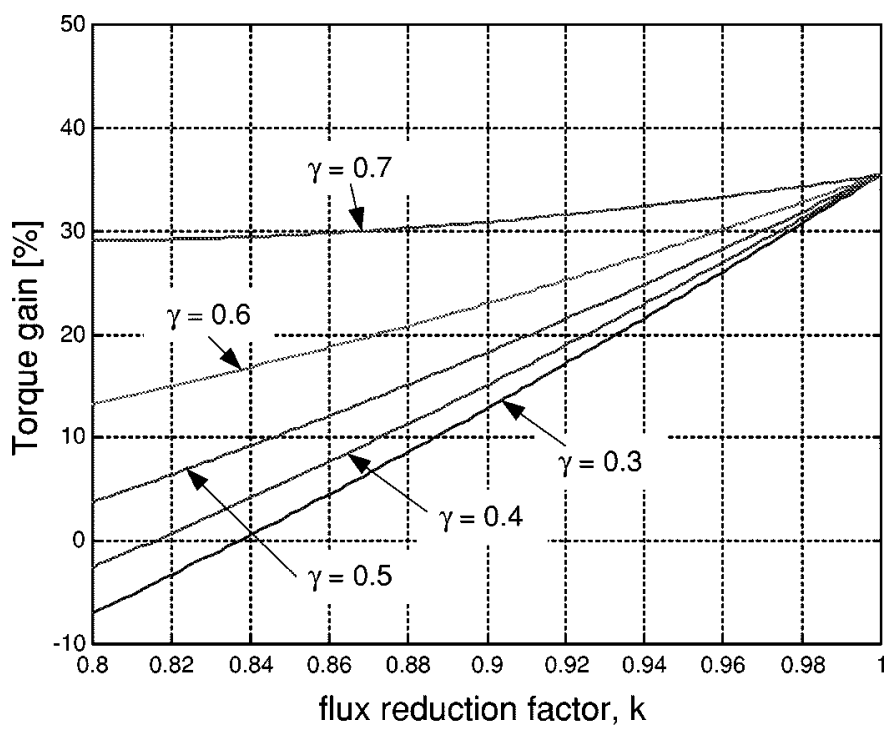

Fig. 2. Torque enhancement considering reduction in the air-gap flux and increase in the surface current density.

Using a similar derivation, an equivalent expression can be found for the baseline machine torque

$$
T_{\text {baseline }}=\frac{3}{2}\left(\frac{P}{2}\right) \frac{\left[\left(\frac{2}{\pi} B_{\max }\right) A_{\text {pole }}\right]^{2}\left(\omega_{e}-\frac{P}{2} \omega_{r m}\right)}{\frac{12}{S_{r}} r_{b e}} .
$$

Taking the ratio the contribution of the third harmonic can be found

$$
\frac{T_{6 \text { phase- } 3 h}}{T_{\text {baseline }}}=\frac{\left[\frac{1}{3 \sqrt{3}}\right]^{2}}{\frac{1}{2}}=\frac{2}{27}=0.0741 .
$$

Also, the contribution of the third harmonic is $7.4 \%$ of the value produced by the baseline machine. The total torque improvement is, then,

$$
\frac{T_{6 \text { phase-fund }}+T_{6 \text { phase- } 3 h}-T_{\text {baseline }}}{T_{\text {baseline }}} 100 \%=40.7 \% .
$$

It must be noted, however, that the peak value stator core flux has not been maintained constant so that the amount of improvement that can actually be realized depends upon the saturation level permitted in the stator core.

Alternatively, rather than maintain the peak air-gap flux constant after addition of the third harmonic, the peak flux density could be reduced by $1.732 / 2$ or 0.866 and the fundamental component kept constant. In this case, the slot area available for copper could be increased permitting a $14 \%$ increase in current and a $14 \%$ increase in torque for the same tooth and core flux density.

It can be shown [15] that the total torque enhancement is a function of the peak air-gap flux, therefore, a function of a flux reduction factor $k$, and the initial tooth-to-slot aspect ratio $\gamma$, which determines the percentage increase allowed for the current density in the machine. Fig. 2 shows the theoretical torque enhancement in the induction machine as a function of these two factors. For a typical 0.5 tooth-to-slot aspect ratio and $87 \%$

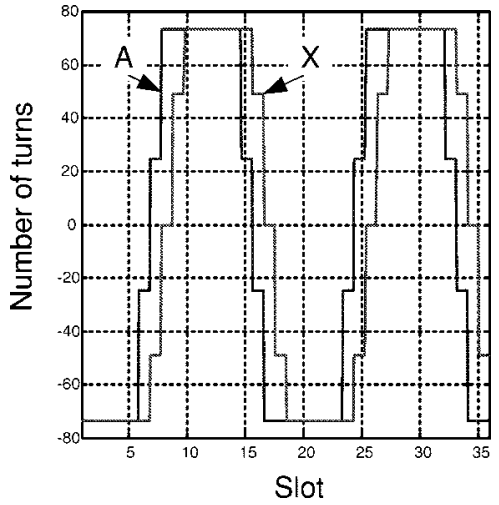

(a)

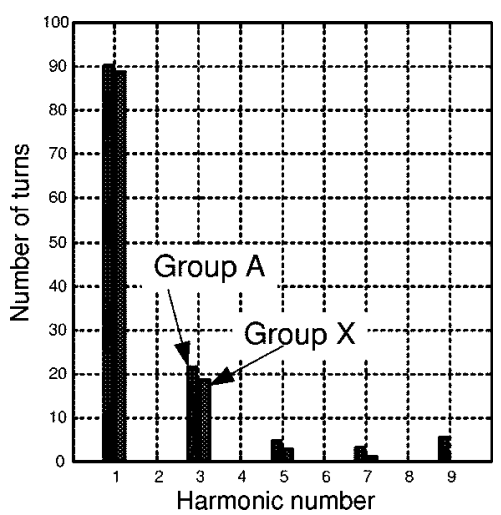

(b)

Fig. 3. Phases $a$ and $x$ winding distribution. (a) Winding function. (b) Harmonic composition.

flux reduction, a torque enhancement of approximately $14 \%$ is obtained, as mentioned before.

\section{MAChine Design}

For verification, an asymmetric six-phase induction motor was designed using a conventional three-phase motor as baseline. The new winding distribution accommodates in the same frame size as the baseline three-phase motor and both machines have similar peak air gap fluxes.

From a single-layer three-phase stator, a double-layer sixphase distribution is implemented by dividing the three phases into two groups that are spatially shifted 30 electrical degrees. Fig. 3 shows the winding functions, and their harmonic composition, for phases $a$ and $x$. The spatial phase shift between the two windings and the presence of the third harmonic component in the distribution necessary to interact to the injected third harmonic currents can be seen.

For the baseline machine, from the nameplate and geometrical data, the air-gap flux is calculated. The stator phase voltage of the machine is calculated as [13]

$$
V_{s f}=\omega_{e} k_{1} N_{s} \Phi_{p}
$$

where

$$
\Phi_{p}=\frac{2 D_{i s} l_{s}}{P} B_{g 1}
$$




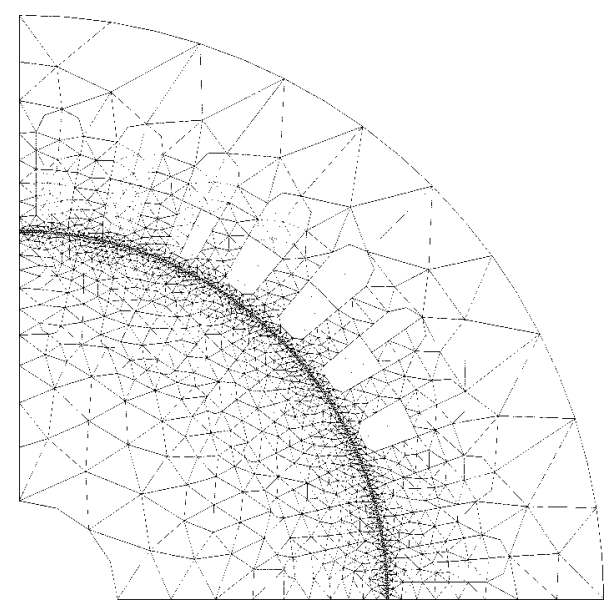

Fig. 4. Finite-element mesh with 6063 nodes and 3006 surfaces.

where $D_{i s}$ is the stator inner diameter, $l_{s}$ the stator length, $P$ the number of poles, and $B_{g 1}$ the peak fundamental air-gap flux. The number of series connected turns per phase is defined as

$$
N_{s}=\frac{\text { (turns/coil)(coil side/slot)(number of slots) }}{\text { (number of phases)(circuits) }} \text {. }
$$

Thus, for a single-layer three-phase machine, one has

$$
N_{s}=\frac{\left(n_{s} / 2\right)(1) S_{1}}{3 C} .
$$

For a $230-\mathrm{V}$ connection with $n_{s}=98$ conductors per slot, $C=4$ circuits, and $S_{1}=36$ stator slots, the number of series-connected turns per phase $N_{s}=147$. Using the physical dimensions of the machine, the peak fundamental air-gap flux density is calculated to be $B_{g 1}=0.57 \mathrm{~T}$ when $60-\mathrm{Hz}$ operation and unit winding factor are considered.

For a six-phase machine with a double-layer winding,

$$
N_{s}=\frac{\left(n_{s} / 4\right)(2) S_{1}}{6 C} .
$$

With the same physical dimensions and same peak fundamental air-gap flux density, the flux per pole $\Phi_{p}$ is the same for the six-phase machine when only the fundamental component is considered. From (26), it is seen that the number of serialconnected turns per phase must be the same for the six-phase machine and, so, for keeping the same $N_{s}$, either $n_{s}$ has to be doubled or $C$ has to be reduced to its half. For this machine configuration, the reduction of $C$ guarantees the accommodation of the conductors in the slots. Finally, the electric loading has to be checked for the new winding configuration. The electric loading for the machine is determined by

$$
K_{s \mathrm{rms}}=\frac{S_{1} n_{s}\left(I_{s} / C\right)}{\sqrt{2} \pi D_{i s}}
$$

with all geometrical variables in (30) constant, the reduction in the number of circuit by half causes the stator current to reduce by half. This is reasonable since now there are two three-phase windings instead of one in the baseline machine.

To measure the flux distribution in the air gap, a full pole pitch search coil [14] was inserted in the machine stator and, with the aid of an integrator, the flux can be determined.

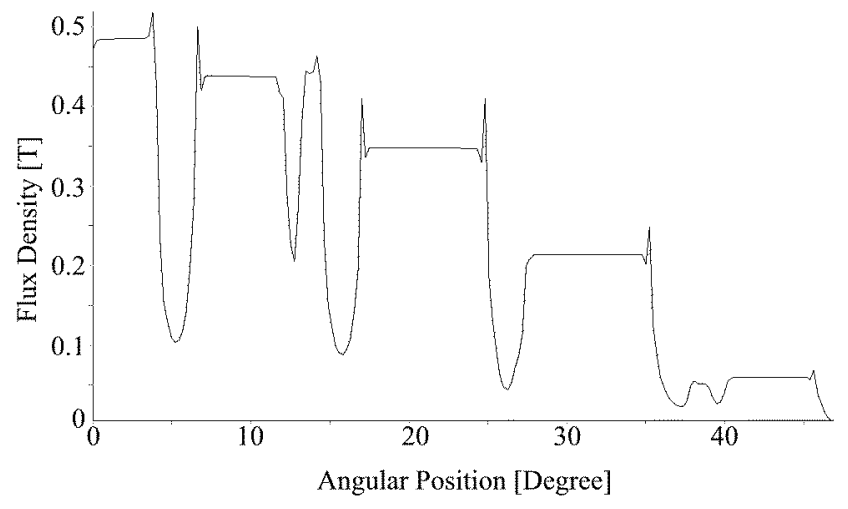

Fig. 5. Air-gap flux density distribution without third harmonic current injection. Peak phase current $I_{\mathrm{pk}}=0.5 \mathrm{~A}$.

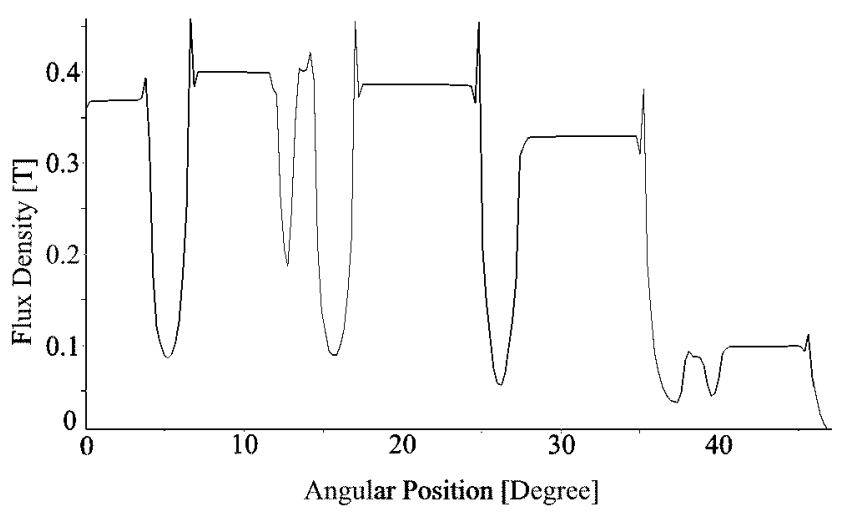

Fig. 6. Air-gap flux density distribution with third harmonic current injection Peak phase current $I_{\mathrm{pk}}=0.5$ A. Peak third harmonic phase current $I_{3 p k}=0.4$ A.

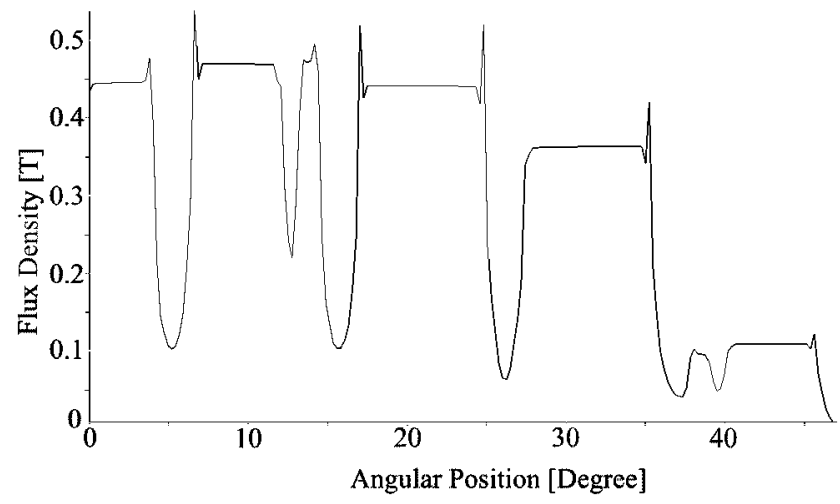

Fig. 7. Air-gap flux density distribution with third harmonic current injection. Peak phase current $I_{\mathrm{pk}}=0.58 \mathrm{~A}$. Peak third harmonic phase current $I_{3 p k}=$ $0.4 \mathrm{~A}$.

\section{FINITE-ELEMENT ANALYSIS}

Finite-element analysis is conducted in the six-phase machine to calculate the air gap flux distribution and to demonstrate the 


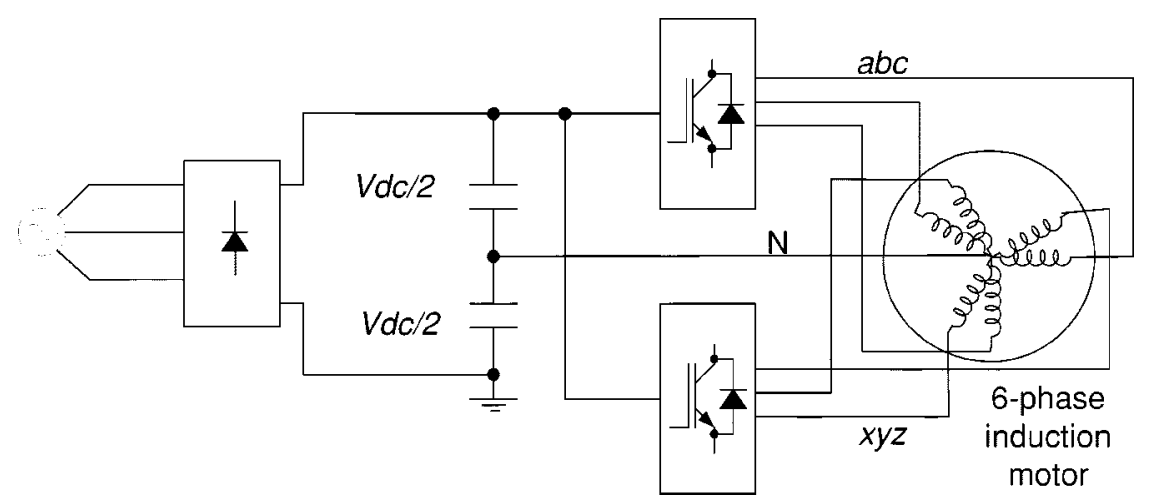

Fig. 8. Six-phase machine drive system.

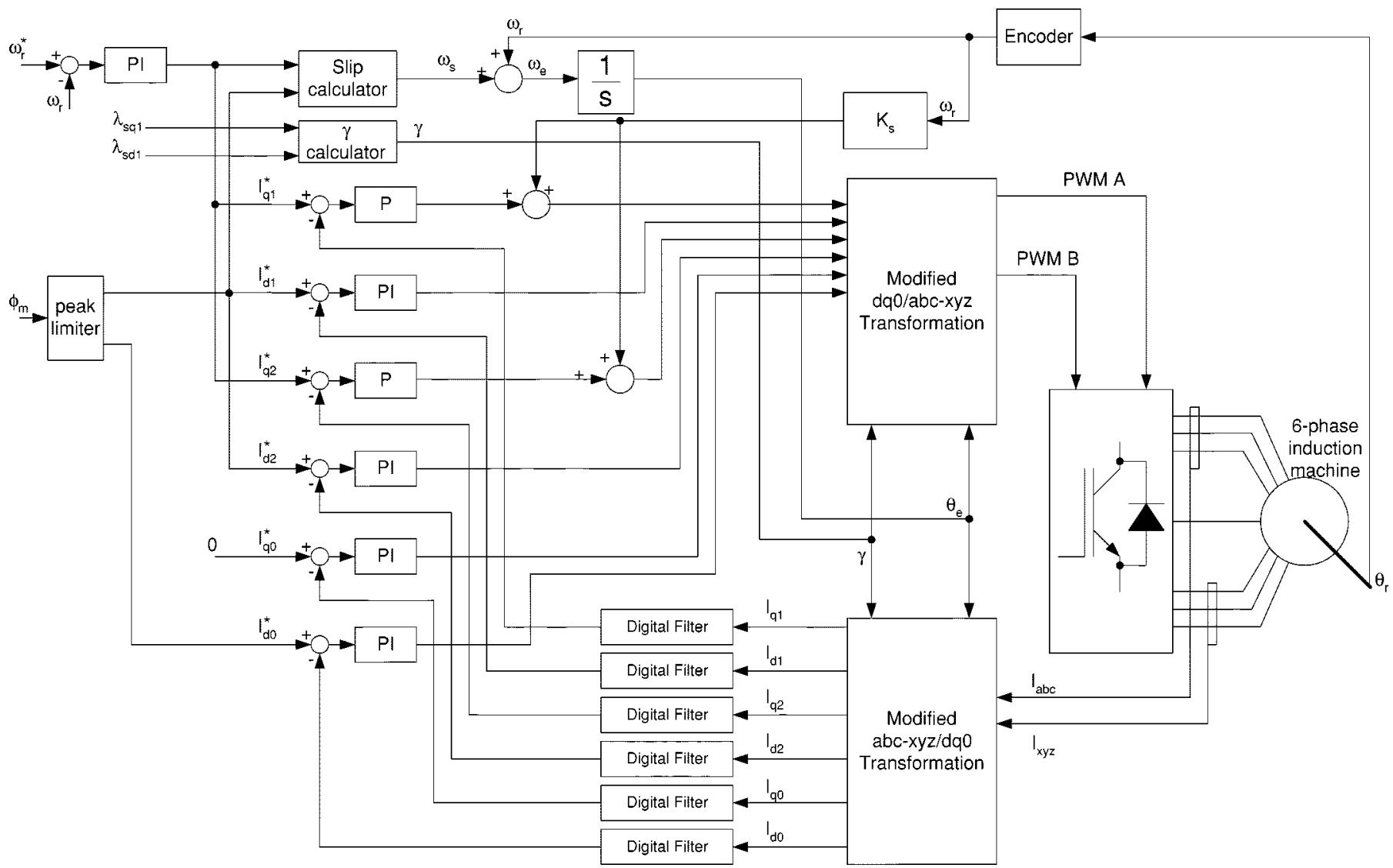

Fig. 9. Speed control block diagram.

increase in the fundamental flux component. Fig. 4 shows the finite-element mesh utilized for the representation of one-quarter of the machine. Boundary condition symmetry is used to obtain the results for the entire machine.

The flux density distribution corresponding to half magnetic pole for the no third harmonic injection and peak phase current $I_{\mathrm{pk}}=0.5 \mathrm{~A}$ is shown in Fig. 5. This current is not enough to drive the machine into saturation and the distribution resembles a sine wave.

The rotor and stator slot openings flux harmonics are also clear on this plot. Injecting third harmonic currents causes the peak flux density to decrease, as can be seen in Fig. 6, where the peak current is kept the same and a third harmonic current $I_{3 p k}=0.4 \mathrm{~A}$ is added. Since the peak flux decreases, the fun- damental current can be increased until the peak flux reaches its previous value. This makes the fundamental flux to increase without sending the peak value beyond its limits. Fig. 7 shows the distribution for $I_{\mathrm{pk}}=0.58 \mathrm{~A}$ and $I_{3 p k}=0.4 \mathrm{~A}$ where a flattened flux distribution is obtained.

\section{SYSTEM DESCRIPTION}

A mathematical model was derived and an experimental setup was built to test the proposed technique. This paper focuses on the experimental results obtained. Fig. 8 shows the setup configuration. A common DC link is used for two three-phase inverters connected to each winding group of the six-phase machine. The neutral is connected to the mid point of the dc-link capacitors. 
An additional inverter leg is provided for connection of the neutral point when necessary. This additional leg is particularly important when operating at low frequencies where the injection of third harmonic currents in the midpoint of the capacitor bank causes undesirable ripple voltages.

A digital signal processor (DSP) card that incorporates two independent three-phase PWM modulators with complementary outputs controls the system. The software was developed in $\mathrm{C}$ language and the control panels were developed using hypertext markup language (HTML) scripting and ActiveX commands. This enables the system control and monitoring over any internal or external network.

To obtain the necessary alignment between fundamental and third harmonic currents, a current-regulated supply must be used. With a voltage supply the alignment is not guaranteed since the machine's impedance changes with operational conditions. Also, it was observed that with a voltage supply (no current regulation) even sharing of the output power between the two inverters is not possible when practical aspects such as differences in the winding functions are considered.

A current-regulated PWM inverter was then implemented and used as the inner loop for a speed controller as shown in Fig. 9. Here the cross-coupling decoupling is omitted but for a highperformance operation, it must be included. A standard proportional plus integral (PI) controller is used to implement an indirect flux control algorithm. The flux peak limiter controls the maximum air-gap flux by injecting third harmonic zero-sequence currents. A modified $a b c-x y z / d q 0$ transformation is used to map the alternate currents into dc quantities in the $d q 0$ domain. This transformation follows the standard $a b c / d q 0$ decomposition, but it is modified to include the third harmonic frequency and also maps third harmonic signals into dc quantities. This approach facilitates the design of the control system.

\section{EXPERIMENTAL RESULTS}

The six-phase machine was tested experimentally to verify the operation with third harmonic current injection. The flux distribution is obtained using search coils installed in the machine and the torque is measured directly in the shaft by means of a torque transducer.

When supplied by a voltage-source inverter, asymmetries in the machine design cause uneven distribution of power between the two three-phase inverters. This feature can be seen in Fig. 10 where the machine is tested at rated voltage and no third harmonic injection. Phases $a$ and $x$ currents are shown together with the air-gap flux distribution. Since phase $a$ current is practically zero, all power is supplied through phase $x$. To correct for this unbalanced operation, a correction angle is applied to the reference voltages to adjust the phase displacement between the two three phase groups. Fig. 11 shows the balanced operation of the system.

The fundamental to third harmonic phase relation determines the air-gap flux waveform. The correct operation of the system requires a flattened flux waveform for all operating points. Fig. 12 shows a typical problem when this phase relation is not observed and the air-gap flux waveform deviates from the ideal

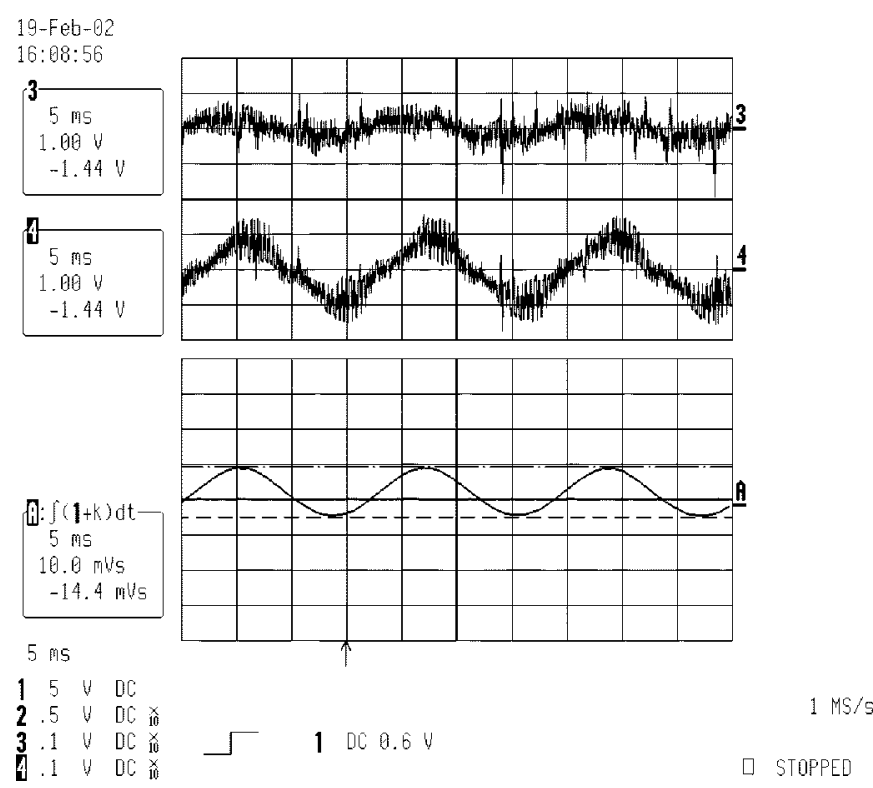

Fig. 10. Unbalanced operation of the six-phase machine at no load. $f=60$ $\mathrm{Hz}, V_{s}=85 \mathrm{~V}$. Trace A: flux density distribution $(0.788 \mathrm{~T} / \mathrm{div})$; trace 3 : phase $a$ current $(3.125 \mathrm{~A} / \mathrm{div})$; trace 4 : phase $x$ current $(3.125 \mathrm{~A} / \mathrm{div})$.

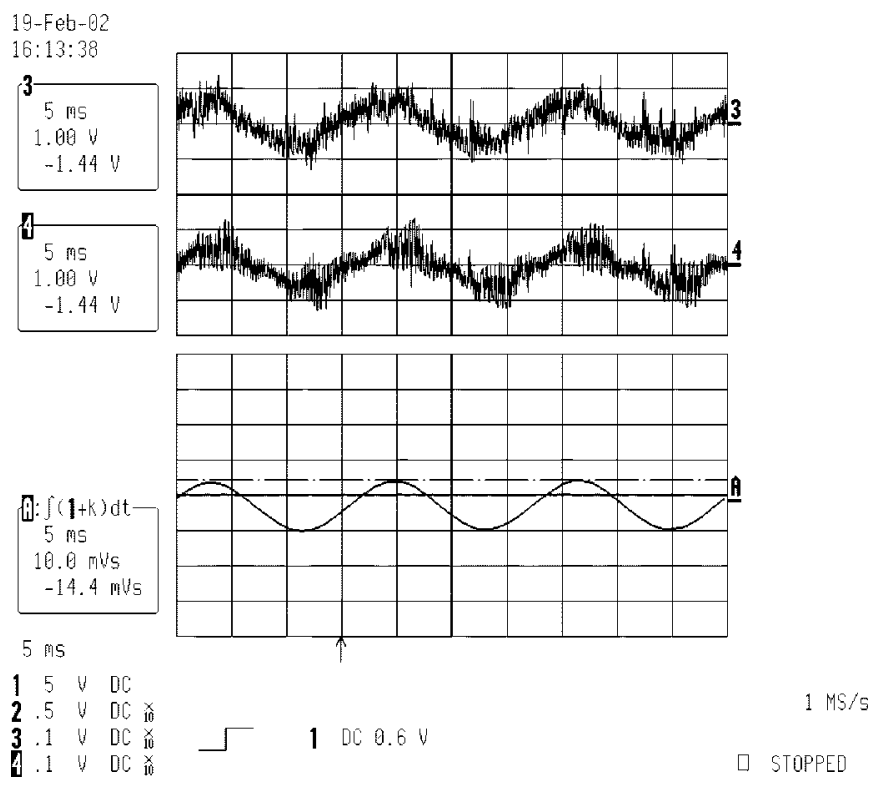

Fig. 11. Balanced operation of the six-phase machine at no load. $f=60 \mathrm{~Hz}$, $V_{s}=85 \mathrm{~V}$. Trace A: flux density distribution $(0.788 \mathrm{~T} / \mathrm{div})$; trace 3 : phase $a$ current (3.125 A/div); trace 4: phase $x$ current (3.125 A/div).

one. For this case, the peaked shaped flux can cause saturation of the stator teeth.

The reshaping of the air-gap flux distribution is demonstrated by performing a no-load test in the machine with a voltagesource inverter. Fig. 13 shows phases $a$ and $x$ currents and the flux density distribution for a peak value of $B_{\mathrm{pk}}=0.82 \mathrm{~T}$ and no third harmonic injection (operation at $f=40 \mathrm{~Hz}$ and $V_{s}=84 \mathrm{~V}$ ). The currents are $30^{\circ}$ phase shifted from each other as necessary for the proposed machine operation. Without the third harmonic currents, the six-phase machine operates similarly to the three-phase baseline machine. Adding third harmonic currents $\left(V_{s 3 h}=14 \mathrm{~V}\right)$ causes the peak flux density to 


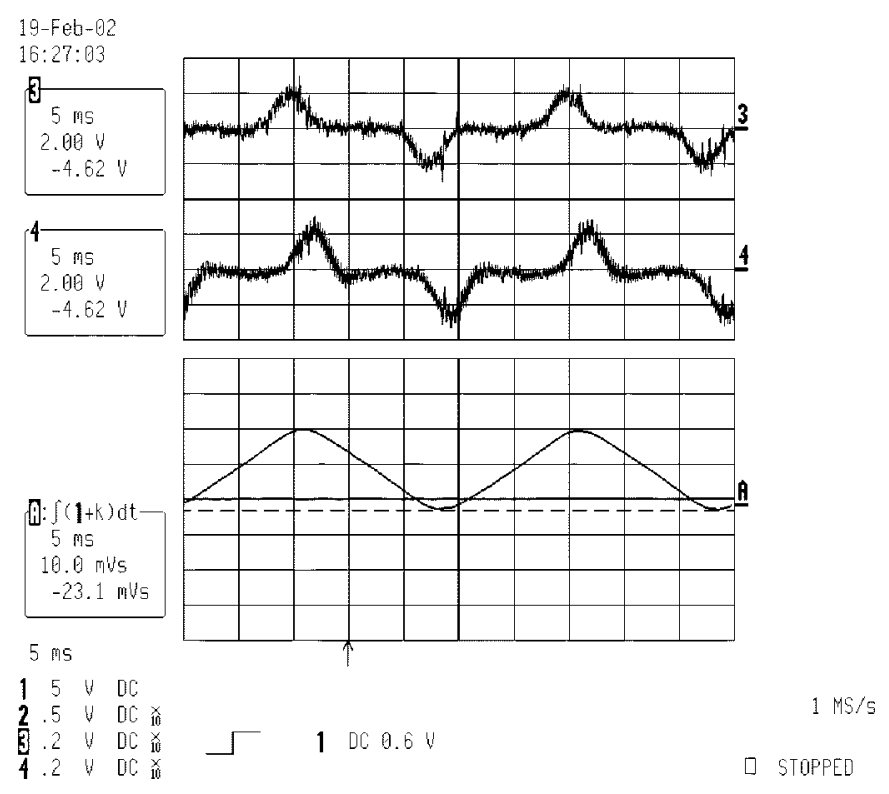

Fig. 12. Incorrect fundamental to third harmonic phase relation. No-load operation at $f=40 \mathrm{~Hz}, V_{s}=88 \mathrm{~V}, V_{s 3 h}=14 \mathrm{~V}$. Trace A: flux density distribution $(0.788 \mathrm{~T} / \mathrm{div})$; trace 3 : phase $a$ current $(6.25 \mathrm{~A} / \mathrm{div})$; trace 4 : phase $x$ current $(6.25 \mathrm{~A} / \mathrm{div})$.

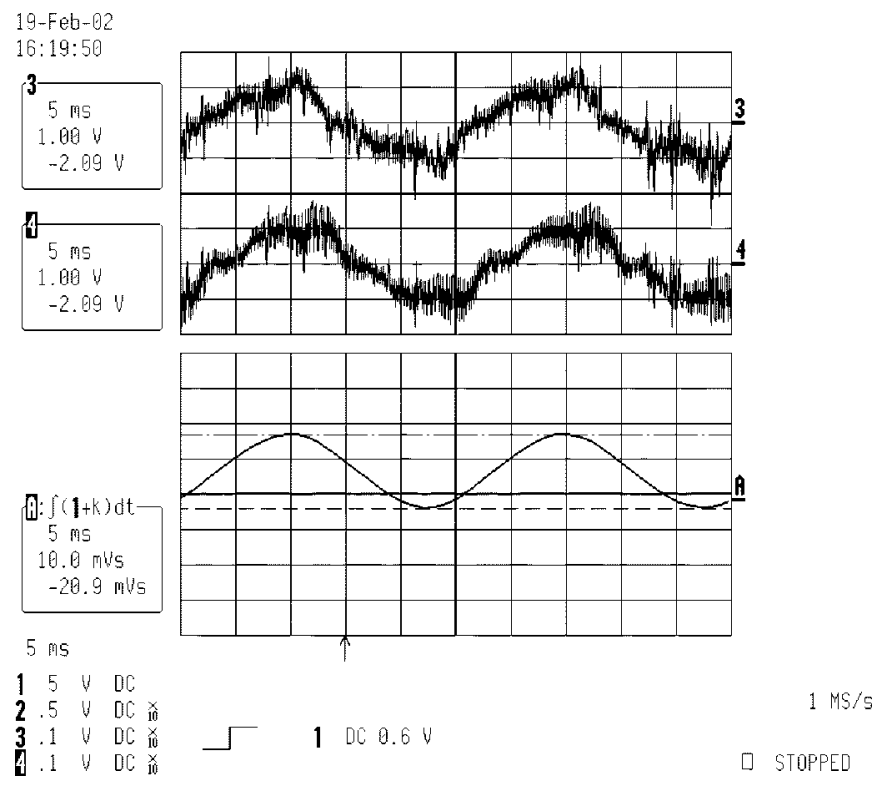

Fig. 13. No-load operation of the six-phase machine without third harmonic injection. $f=40 \mathrm{~Hz}, V_{s}=84 \mathrm{~V}$. Trace A: flux density distribution $(0.788$ T/div); trace 3: phase $a$ current $(3.125 \mathrm{~A} / \mathrm{div})$; trace 4 : phase $x$ current $(3.125$ $\mathrm{A} / \mathrm{div})$.

decrease to $B_{\mathrm{pk}}=0.74 \mathrm{~T}$, as seen in Fig. 14 . This corresponds to a subutilization of the machine iron when compared to the previous case. To raise the peak flux, the fundamental current can be increased until the previous level is reached. This result is shown in Fig. 15 where the peak flux correction is applied and the peak levels with the no third harmonic case.

For operation at $f=45 \mathrm{~Hz}$, Fig. 16 shows the torque $x$ speed characteristic for the prototype machine ( $3 \mathrm{hp}$ ) when compared to the baseline three-phase machine. It is clear the enhancement in the torque production. However, the increased torque

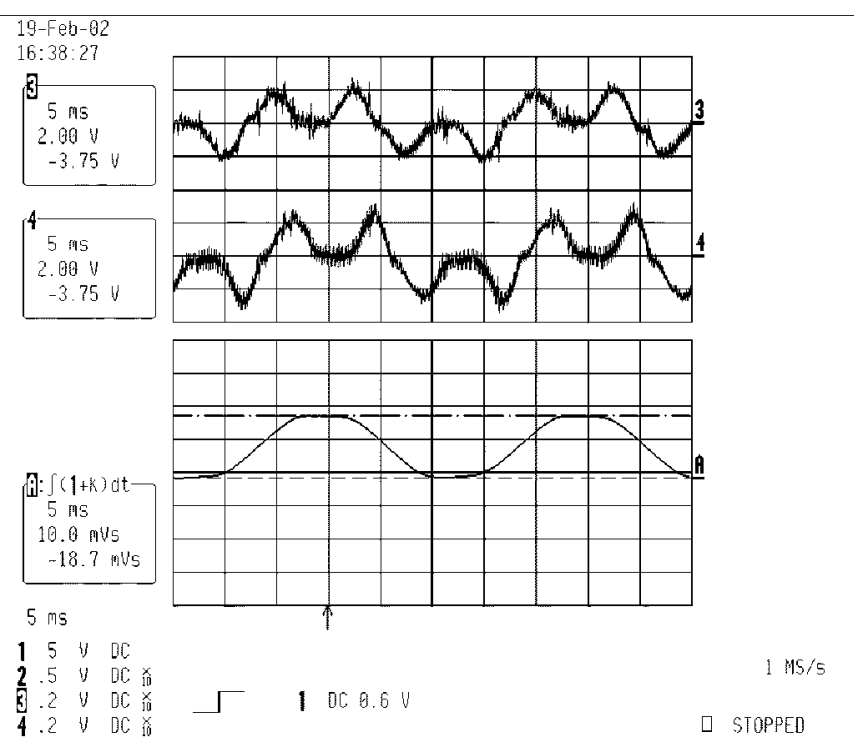

Fig. 14. No-load operation of the six-phase machine with third harmonic injection. $f=40 \mathrm{~Hz}, V_{s}=84 \mathrm{~V}, V_{s 3 h_{t}}=14 \mathrm{~V}$. Trace A: flux density distribution ( $0.788 \mathrm{~T} / \mathrm{div})$; trace 3 : phase $a$ current $(6.25 \mathrm{~A} / \mathrm{div})$; trace 4 : phase $x$ current $(6.25 \mathrm{~A} / \mathrm{div})$.

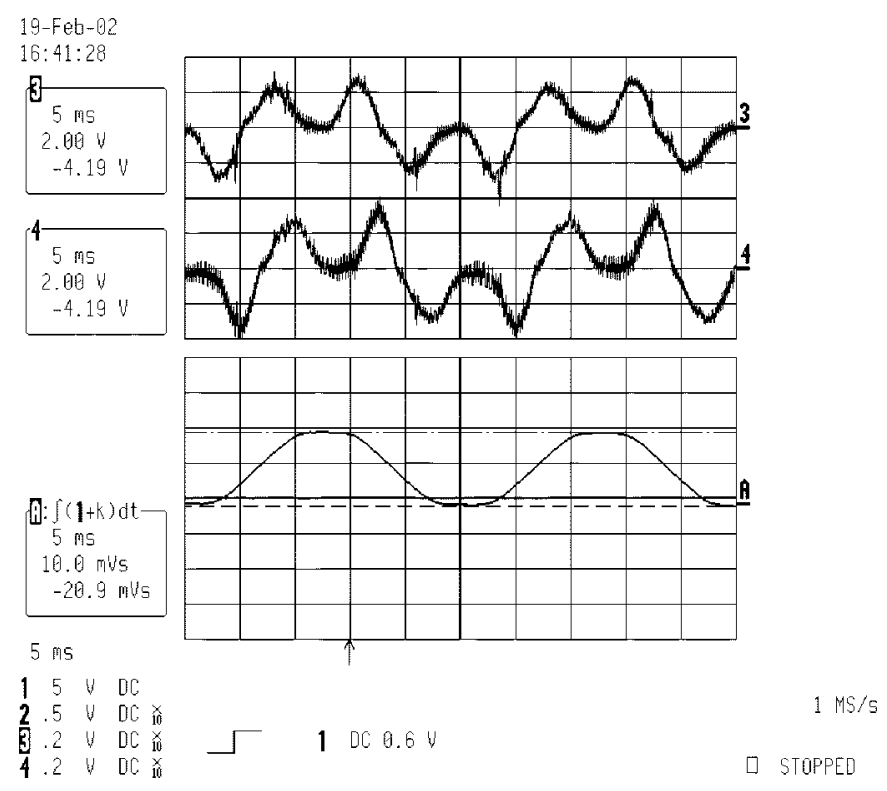

Fig. 15. No-load operation of the six-phase machine with third harmonic injection. $f=40 \mathrm{~Hz}, V_{s}=93 \mathrm{~V}, V_{s 3 h_{t}}=14 \mathrm{~V}$. Trace A: flux density distribution ( $0.788 \mathrm{~T} / \mathrm{div})$; trace 3 : phase $a$ current $(6.25 \mathrm{~A} / \mathrm{div})$; trace 4 : phase $x$ current $(6.25 \mathrm{~A} / \mathrm{div})$.

is obtained by increasing the currents in the machine and by reshaping its air-gap flux. Both factors contribute for changes in the losses that have to be accounted for. Fig. 17 shows the measured magnetic losses at $f=40 \mathrm{~Hz}$ for the six-phase machine, compared to the baseline machine. It can be seen that the magnetic losses do not increase significantly in the rated flux range. However, as shown in Fig. 18, the copper loss increase is not negligible, especially in a low-power machine. This has big impact on the efficiency of the machine and is more noticeable at lower mechanical load. Fig. 19 show the measured efficiency for $f=45 \mathrm{~Hz}$ operation. The proposed system presents similar efficiency to the baseline machine only for higher load conditions. 


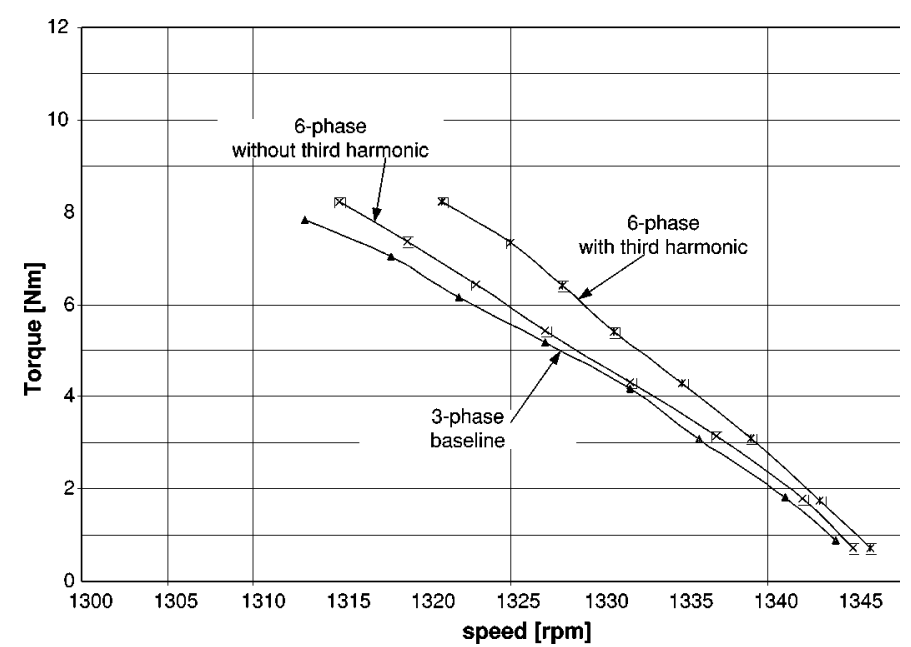

Fig. 16. Torque $x$ speed characteristics for 3-hp six-phase machine. Six-phase machine operating with and without third harmonic currents, compared to the baseline three-phase machine.

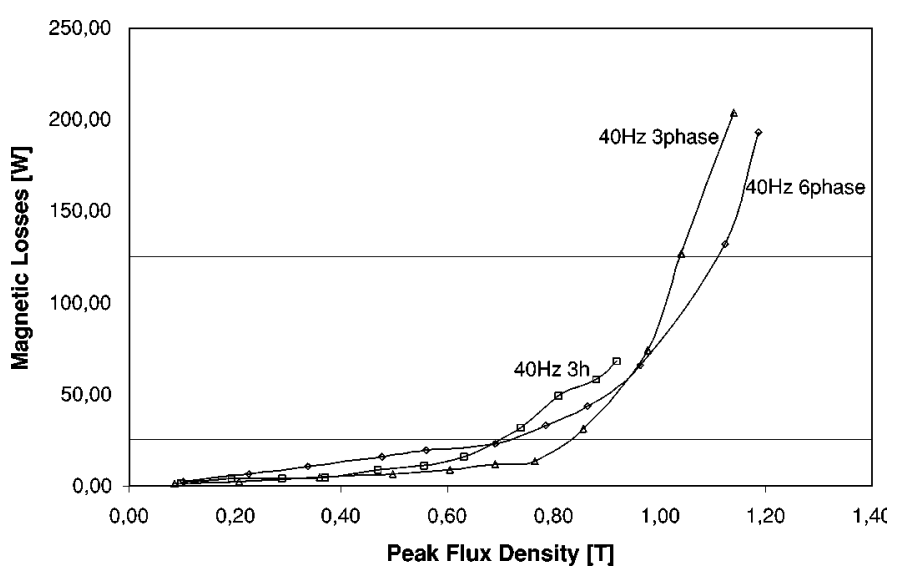

Fig. 17. Magnetic losses at $40 \mathrm{~Hz}$. Comparison between the 3-hp $\left(P_{R}=\right.$ $1400 \mathrm{~W}$ ) six-phase machine with and without third harmonic injection and the baseline three-phase machine.

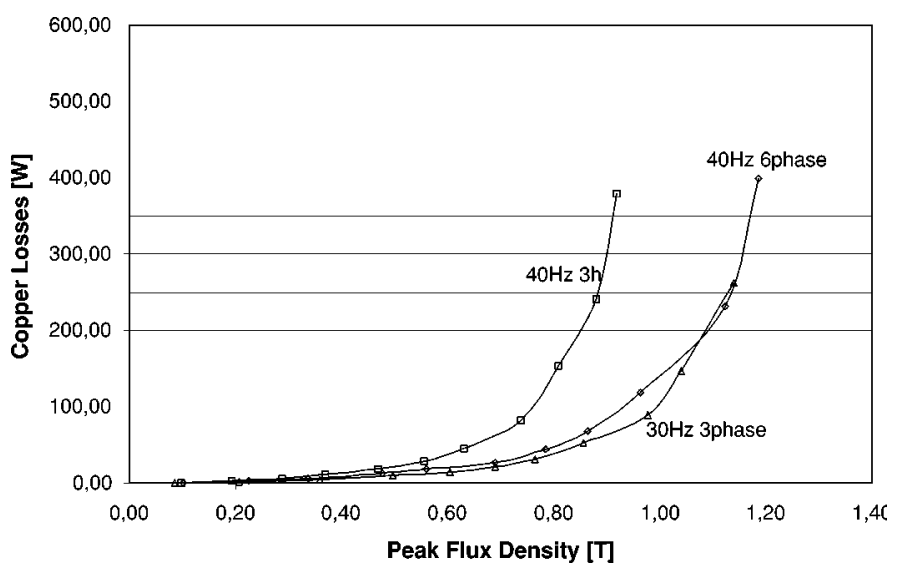

Fig. 18. Stator copper losses at $40 \mathrm{~Hz}$. Comparison between the 3 -hp $\left(P_{R}=\right.$ $1400 \mathrm{~W}$ ) six-phase machine with and without third harmonic injection and the baseline three-phase machine.

Therefore, the proposed system operates without third harmonic current injection until a load close or beyond the rated torque is applied.

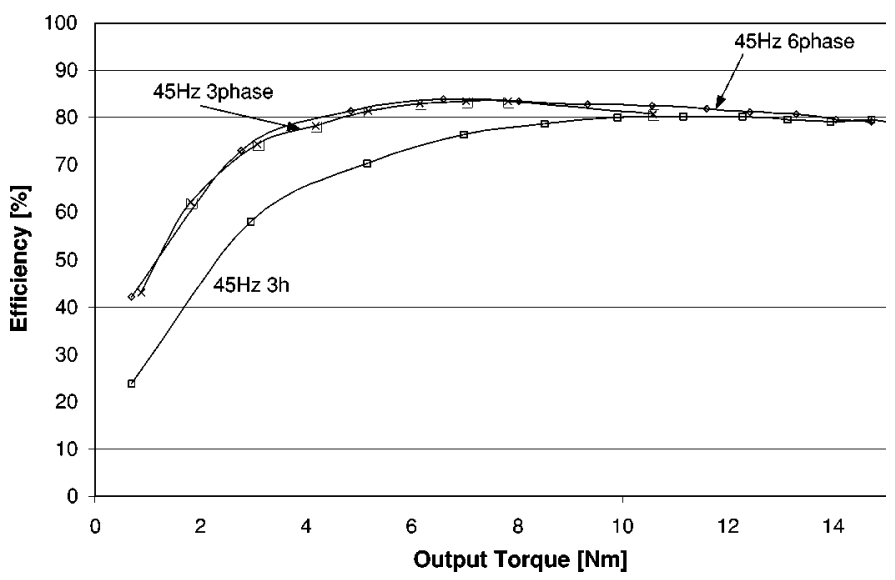

Fig. 19. Six-phase machine efficiency at $45 \mathrm{~Hz}$. Comparison between the six-phase machine with and without third harmonic injection and the baseline three-phase machine.

\section{CONCLUSION}

Multiphase systems are broadly used in industry to achieve higher power levels based in limited-range power converters. Additional torque production can be obtained in these systems if third harmonic currents are injected so as to reshape the flux distribution in the machine and, therefore, obtain higher fundamental flux densities without extrapolating the flux limits and forcing the machine under saturation.

This paper has proposed the use of a six-phase induction machine to gain these benefits. By constructing the machine with two three-phase groups phase shifted by $30^{\circ}$, it is possible to create an additional rotating field in synchronism with the fundamental by using third harmonic zero-sequence currents. In so doing, the air-gap flux presents a third harmonic component that reduces the peak flux density. Additional torque is then obtained by increasing the fundamental flux component in such a manner to reestablish the air-gap flux to its original peak value and by the extra third harmonic rotating field.

Through analytical analysis, assuming saturation of the stator teeth as the constraint on flux density, it has been shown that an increase of up to $40 \%$ in the torque production can be expected with this technique when compared to a standard three-phase machine, for the same peak flux distribution. Alternatively, if both tooth and core flux density are to be constrained to fixed values at least a $14 \%+7 \%=21 \%$ increase is possible by enlarging the stator slot area. To obtain the benefits, the machine's winding function has to include third harmonic components. This is true for most of electrical machined due to the discrete construction of the windings that are distributed in finite number of slots. The best improvement would be obtained using a concentrated winding machine since for this case the higher third harmonic component in the winding function is obtained.

Finite-element analysis has been used to show the behavior of air-gap and core flux with third harmonic currents. This analysis is particularly important to correctly map the behavior of the flux in areas too complicated to have a direct measurement.

A fundamental issue in this application is the necessity of controlling the phase relation between fundamental and third harmonic flux components. If this relation is lost, a peaked flux density could be generated instead of the proposed flattened one. 
Current control is, therefore, necessary for this application and a control system is proposed where a modified $a b c / d q 0$, now called $a b c-x y z / d q 0$, synchronous frame transformation is developed to include the third harmonic components. This transformation maps all currents into a $d q 0$ domain as de quantities what facilitates the control design and tuning.

The proposed system was tested experimentally to show the expected torque improvement. It is shown that for voltage fed systems the current sharing between the two three-phase groups can be uneven causing problems for both machine and power electronics. The experimental results show that for same air gap peak flux density, the torque can be increased with the third harmonic current injection as a result of the increase in the fundamental component of current and flux. An experimental assessment of magnetic and copper losses is presented and used to define the operation strategy for the proposed system.

\section{REFERENCES}

[1] A. R. Bakhshai, G. Joos, and H. Jin, "Space vector PWM control of a split-phase induction machine using the vector classification technique," in Proc. IEEE APEC'98, vol. 2, Feb. 1998, pp. 802-808.

[2] R. H. Nelson and P. C. Krause, "Induction machine analysis for arbitrary displacement between multiple windings," IEEE Trans. Power App. Syst., vol. PAS-93, pp. 841-848, May 1974.

[3] L. Xu and L. Ye, "Analysis of a novel stator winding structure minimizing harmonic current and torque ripple for dual six-step converter-fed high power ac machines," IEEE Trans. Ind. Applicat., vol. 31, pp. 84-90, Jan./Feb. 1995.

[4] A. R. Munoz and T. A. Lipo, "Dual stator winding induction machine drive," IEEE Trans. Ind. Applicat., vol. 36, pp. 1369-1379, Sept./Oct. 2000.

[5] O. Ojo and I. E. Davidson, "PWM-VSI inverter-assisted stand-alone dual stator winding induction generator," IEEE Trans. Ind. Applicat., vol. 36, pp. 1604-1611, Nov./Dec. 2000.

[6] G. Oriti, L. Julian, and T. A. Lipo, "An inverter/motor drive with common mode voltage elimination," in Conf. Rec. IEEE-IAS Annu. Meeting, vol. 1, Oct. 1997, pp. 587-592.

[7] T. A. Lipo, "A $d-q$ model for six phase induction machines," in Proc. ICEM'80, Athens, Greece, Sept. 1980, pp. 860-867.

[8] J. Fu and T. A. Lipo, "Disturbance free operation of a multiphase current regulated motor drive with an opened phase," IEEE Trans. Ind. Applicat., vol. 30, pp. 1267-1274, Sept./Oct. 1994.

[9] H. A. Toliyat, T. A. Lipo, and J. C. White, "Analysis of a concentrated winding induction machine for adjustable speed drive applications. I. Motor analysis," IEEE Trans. Energy Conversion, vol. 6, pp. 679-683, Dec. 1991.

[10] — "Analysis of a concentrated winding induction machine for adjustable speed drive applications. II. Motor design and performance," IEEE Trans. Energy Conversion, vol. 6, pp. 684-692, Dec. 1991.
[11] S.-S. P. Liou, "Theoretical and experimental study of polyphase induction motors with added third harmonic excitation," Master's thesis, Univ. Texas, Austin, 1985.

[12] N. L. Schmitz and D. W. Novotny, Introductory Electromechanics. New York: Ronald, 1965.

[13] T. A. Lipo, Introduction to AC Machine Design. Madison, WI: Univ. of Wisconsin Press, 1996.

[14] - "Flux sensing and control of static AC drives by use of flux coils," IEEE Trans. Magn., vol. MAG-13, pp. 1403-1408, Sept. 1997.

[15] R. Lyra, "Torque density improvement in a six-phase induction motor with third harmonic current injection," Ph.D. dissertation, Univ. Wisconsin, Madison, 2002.

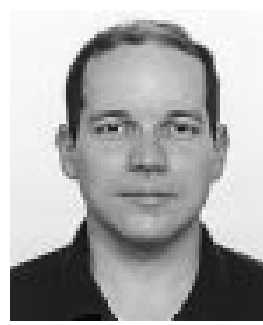

Renato O. C. Lyra (S'98-M'02) was born in Belo Horizonte, Brazil. He received the B.E.E. and M.E.E degrees in electrical engineering from the Universidade Federal de Minas Gerais, Belo Horizonte, Brazil, in 1991 and 1994, respectively, and the Ph.D. degree from the University of Wisconsin, Madison, in 2002 .

Since 1994, he has been with the Department of Electrical Engineering, Universidade Federal de Minas Gerais, as an Assistant Professor. His primary areas of interest are electrical machines design, motor drives, and renewable energy systems.

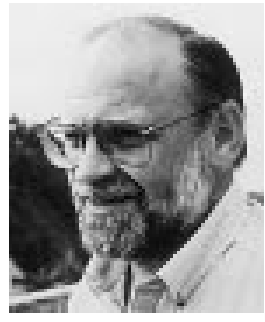

Thomas A. Lipo (M'64-SM'71-F'87) is a native of Milwaukee, WI. He received the B.E.E. and M.S.E.E. degrees from Marquette University, Milwaukee, WI, in 1962 and 1964, respectively, and the Ph.D. degree in electrical engineering from the University of Wisconsin, Madison, in 1968.

From 1969 to 1979, he was an Electrical Engineer in the Power Electronics Laboratory of Corporate Research and Development, General Electric Company, Schenectady, NY. He became a Professor of Electrical Engineering at Purdue University, West Lafayette, IN, in 1979 and, in 1981, he joined the University of Wisconsin, Madison, in the same capacity. He is presently the W.W. Grainger Professor for Power Electronics and Electrical Machines, Co-Director of the Wisconsin Electric Machines and Power Electronics Consortium, and Director of the Wisconsin Power Electronics Research Center.

Dr. Lipo has received the Outstanding Achievement Award from the IEEE Industry Applications Society, the William E. Newell Field Award from the IEEE Power Electronics Society, and the 1995 Nicola Tesla IEEE Field Award from the IEEE Power Engineering Society for his work. Over the past 30 years, he has served the IEEE in numerous capacities, including President of the IEEE Industry Applications Society. 